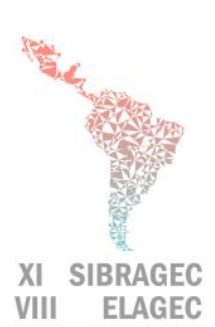

VIII ELAGEC

\section{SIMPÓSIO BRASILEIRO DE GESTÃO E ECONOMIA DA CONSTRUÇÃO \\ VIII ENCUENTRO LATINOAMERICANO DE GESTIÓN Y ECONOMÍA DE LA CONSTRUCCIÓN}

Del conocimiento a la acción: prácticas avanzadas de gestión de la producción

Londrina, Paraná, Brasil. 23 al 25 de Octubre del 2019

\title{
PLATAFORMA DE DATOS ABIERTOS DE CONTRATACION DE OBRAS PÚBLICAS DEL GOBIERNO COLOMBIANO
}

\author{
GOMEZ CABRERA, Adriana (1); PONZ-TIENDA, José Luis (2); PELLICER, \\ Eugenio (3), TORRES, Andrés (4)
}

(1) Pontificia Universidad Javeriana - Facultad de Ingeniería, Universidad de los Andes - Facultad de Ingeniería, teléfono: +(571) 3394949 e-mail: ca.gomez1@uniandes.edu.co (2) Universidad de los Andes - Facultad de Ingeniería, e-mail: jl.ponz@uniandes.edu.co, (3) Universitat Politècnica de València E.T.S.I. Caminos, Canales y Puertos, e-mail: pellicer@upv.es (4) Pontificia Universidad Javeriana Facultad de Ingeniería, e-mail: andres.torres@javeriana.edu.co

\begin{abstract}
In recent years, transparency in public procurement has increased considerably as a strategy to curb corruption and implement best practices by governments. Since 2007, Colombia has been implementing different approaches in this sense, which have been evaluated by international bodies with outstanding results. The country's open data platform is mandatory, and all public entities must publish the processes carried out there. This paper presents the "Sistema Electrónico de Contratación Pública de Colombia SECOP" with emphasis on the construction phase of infrastructure projects, which contains information on all stages of construction projects. There are examples of data and analysis that can be made from them.
\end{abstract}

Keywords: Colombia, contracting, open data, public works.

\section{INTRODUCCIÓN}

La transparencia en torno al gasto público se ha incrementado considerablemente en los últimos años, como respuesta a la necesidad de aumentar la competitividad, integridad y eficiencia del proceso de los procesos de contratación (OPEN CONTRACTING PARTNERSHIP, 2019). Diferentes países a nivel mundial ya cuentan con plataformas que, a partir de datos abiertos, reportan la ejecución del gasto público, situación que ha permitido a investigadores, realizar análisis y encontrar tendencias a partir de las mismas. La literatura reporta que la que la transparencia en la publicación del gasto público, puede dar lugar a un aumento del número de licitadores, la prevalencia de ganadores no locales y la calidad de la ejecución de los contratos (FAZEKAS; TÓTH; KING, 2016). Otros estudios proponen indicadores de corrupción en la contratación pública en Europa, identificando "señales de alarma" que podrían ser indicativas de corrupción como el número de proponentes, la transparencia y duración del proceso y de la evaluación del mismo (FAZEKAS; KOCSIS, 2017).

GOMEZ CABRERA, A.; PONZ-TIENDA, J. L.; PELLICER, E.; TORRES, A. Plataforma de datos abiertos de contratacion de obras públicas del gobierno colombiano. In: SIMPÓSIO BRASILEIRO DE GESTÃO E ECONOMIA DA CONSTRUÇÃO, 11., 2019, Londrina. Anais [...]. Porto Alegre: ANTAC, 2019. Disponível em: https://www.antaceventos.net.br/index.php/sibragec/sibragec2019/paper/view/393 
Respecto a las etapas de los proyectos de construcción más susceptibles a la corrupción, (OWUSU et al., 2019) reportan como criticas la ejecución del proyecto, la precalificación y las etapas de licitación, por esto los sistemas de compra publica pueden facilitar la identificación de tendencias útiles para el desarrollo e implementación de medidas anticorrupción dirigidas a abordar la corrupción en la cadena de suministro de la construcción.

Colombia es un país que en materia de corrupción no obtiene los mejores resultados; el Índice Global de Corrupción calculado por transparencia internacional lo ubica en el puesto 99 entre 180 países con un puntaje de 36/100 (TRANSPARENCY INTERNATIONAL, 2019). Es por esto que el fortalecimiento del sistema de compra pública es fundamental en la lucha contra la corrupción, pues corresponde a más de un tercio del Presupuesto General y entre el 10 y el 15\% del Producto Interno Bruto en el país (ZULETA; SAAVEDRA; MEDELLÍN, 2018).

Aunque los indicadores en materia de corrupción no son alentadores, la transparencia en los procesos de contratación ha sido tema fundamental de los últimos gobiernos y los resultados de la disponibilidad de datos abiertos han sido reconocidos a nivel internacional, obteniendo destacadas posiciones en rankings y evaluaciones. Desde el año 2007 se creó el sistema electrónico para la contratación pública y posteriormente, se creó la Agencia Nacional de Contratación Pública - Colombia Compra Eficiente. Ésta es la entidad encargada actualmente de las plataformas Sistema Electrónico de Contratación Pública - SECOP donde las entidades públicas reportan la totalidad de los procesos de compra pública en el país (COLOMBIA COMPRA EFICIENTE, 2018). En este trabajo, se presentará el Sistema de Compra Pública en Colombia, enfocada a la fase de construcción de los proyectos de infraestructura para dar a conocer su estructura, cantidad y calidad de datos y también se incluirán ejemplos del tipo de análisis que pueden realizarse a partir de los mismos. Se presentarán también los resultados del país respecto a las diferentes iniciativas de medición de datos abiertos.

\section{METODOLOGÍA}

\subsection{Iniciativas internacionales para medición de datos abiertos.}

En esta primera etapa se realizó una recopilación de información sobre las diferentes iniciativas que se han implementado con el fin de medir la transparencia y disponibilidad de información frente a la compra pública por parte de los gobiernos. Se identificaron los resultados obtenidos por Colombia.

\subsection{Análisis del sistema de compra pública de Colombia}

En esta segunda etapa se identificaron el tipo de datos disponibles en la plataforma de datos abiertos de Colombia, respecto a proyectos de construcción, en la cual se encuentra información sobre todas las etapas de los proyectos y sus características. Posteriormente, se realiza un análisis de datos enfocado en procesos de construcción de infraestructura, se presentan recomendaciones sobre la calidad de los datos. 
SIBRAGEC - ELAGEC 2019 - del 23 al 25 de Octubre - LONDRINA - PR

\section{RESULTADOS}

\subsection{Plataforma de datos públicos de Colombia desde la perspectiva de iniciativas internacionales.}

A nivel internacional se han establecido diferentes modelos que definen como deben ser los datos abiertos publicados por los gobiernos. Uno de estos es "Open Data Charter", que propone un conjunto de principios y buenas prácticas para la liberación de datos abiertos gubernamentales estableciendo que los datos deben ser abiertos por defecto, oportunos y exhaustivos, accesibles y utilizables, comparables e interoperables y deben mejorar la gobernanza y la participación ciudadana así como el desarrollo incluyente y la innovación. Este modelo ha sido adoptado por 69 países y gobiernos locales incluyendo Colombia, pero actualmente no hace una calificación de los datos publicados. (OPEN DATA CHARTER, 2019)

Otra iniciativa es el Estándar de Datos para la Contratación Abierta, creada por la organización "Open Contracting Partnership", creado en 2014. Este permite que los usuarios y los socios de todo el mundo publiquen datos legibles por máquinas que se puedan compartir y reutilizar, con el objetivo de analizarlos. Colombia ha adoptado también este estándar, siendo parte los aproximadamente 20 países pioneros, Uruguay, Chile y Paraguay son los países de Latinoamérica que también lo han adoptado (OCP, 2019).

Adicionalmente, se han desarrollado propuestas para medir la calidad de estos datos. Una de estas es el "Global Open Data Index", punto de referencia mundial anual para la publicación de datos de gobierno abierto. Uno de sus indicadores se refiere a "procurement" y mide la competitividad de los procesos por entidad, evalúa que los datos sean de licencia abierta, estén en formato legible por máquina, sean descargables, estén actualizados, disponibles públicamente y de manera gratuita (ZULETA, 2017). Colombia cumple con el $100 \%$ de estos indicadores haciendo parte de los 10 países que a nivel mundial lo hacen. A nivel de Latinoamérica están Méjico, Paraguay y Uruguay (OPEN KNOWLEDGE INTERNATIONAL, 2019). 
SIBRAGEC - ELAGEC 2019 - del 23 al 25 de Octubre - LONDRINA - PR

Figura 1 - Resultados Open Data Barometer

\begin{tabular}{|c|c|c|c|c|}
\hline Country & \begin{tabular}{|l|} 
Score 9 \\
otrof ico
\end{tabular} & 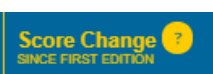 & 。 & \\
\hline (\$) $\begin{array}{c}\text { Canada } \\
\text { See detalis }\end{array}$ & 76 & & 18 & $\Delta$ \\
\hline 我 United Kingdom & 76 & & -4 & $\checkmark$ \\
\hline Australia & 75 & & 17 & $\Delta$ \\
\hline (1) $\begin{array}{c}\text { France } \\
\text { See ofeals }\end{array}$ & 72 & & 17 & $\Delta$ \\
\hline (c): Korea & 72 & & 25 & $\Delta$ \\
\hline$\Leftrightarrow \begin{array}{c}\text { Mexico } \\
\text { see detalls }\end{array}$ & 69 & & 33 & 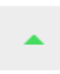 \\
\hline - Japan $_{\text {see detals }}$ & 68 & & 24 & $\Delta$ \\
\hline Nee detalis & 68 & & 5 & $\Delta$ \\
\hline 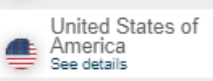 & 64 & & -11 & $v$ \\
\hline - Germany & 58 & & 2 & $\Delta$ \\
\hline 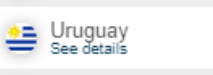 & 56 & & 23 & $\Delta$ \\
\hline - $\begin{array}{c}\text { Colombia } \\
\text { See detalis }\end{array}$ & 52 & & 25 & $\Delta$ \\
\hline
\end{tabular}

Fuente: (WORLD WIDE WEB FOUNDATION, 2017)

Finalmente está "Open Data Barometer" que mide la apertura y la posibilidad de uso de los datos de contratos públicos (WORLD WIDE WEB FOUNDATION, 2017). En su última medición se incluyeron solamente los 30 gobiernos que se han comprometido públicamente a adoptar los Principios de "Open Data Charter". Colombia obtuvo un puntaje de 52/100 (siendo el más alto 76) en esta iniciativa y junto con Uruguay y Méjico representan a los países de Latinoamérica líderes en esta iniciativa. La Figura 1, incluye los países con los puntajes más altos, según esta iniciativa.

Otra propuesta de medición es la desarrollada por parte del "Corruption Research Center Budapest", que analiza las prácticas de publicación de datos de 112 países (FÖLSZ et al., 2018). En esta iniciativa Colombia hace parte del 82\% de los países que tienen una página web para publicar datos de procesos de compra publica, del $56 \%$ publican datos estructurados de contratos adjudicados y del $32 \%$ que los hace descargables en línea. Identifican 20 características como importantes para identificar el riesgo de corrupción y otras anomalías. Ucrania está en primer lugar con disponibilidad 20/20 de las características, seguido por Colombia con 17/20. Los tres aspectos faltantes corresponden al número de proponentes que se presentan a cada proceso y sus datos. Colombia, ocupan el primer lugar en número de filtros disponibles (11) para el análisis de datos y respecto al volumen de datos disponibles (6 millones) ocupa el tercer lugar, después de Estados Unidos (20 millones) y Rusia (17 millones) (FÖLSZ et al., 2018).

\subsection{Plataforma SECOP}

\subsubsection{Características}

La plataforma de datos abiertos de Colombia cuenta con cinco fuentes de datos (Ver

Figura 2) que están disponibles en el portal del Ministerio de Tecnologías de Información y Comunicaciones: Datos Abiertos (www.datos.gov.co - Entidad Agencia 
SIBRAGEC - ELAGEC 2019 - del 23 al 25 de Octubre - LONDRINA - PR

Nacional de Contratación Pública "Colombia Compra Eficiente"). Este trabajo se centra en SECOP I y II que son las que incluyen datos de contratación de proyectos de construcción y que son actualizadas diariamente.

Figura 2 - Fuentes de datos en la plataforma de compra pública.

\section{SECOP I}

- Primera versión implementada desde el año 2011 y aun vigente para entidades territoriales.

\section{SECOP II}

- Segunda versión implementada desde 2015. Es una plataforma transaccional donde las entidades estatales, pueden hacer contratación en línea. Todas las entidades públicas migrando actualmente a esta plataforma.

\section{Plan Anual de Adquisiciones}

- Grupo de datos con la información consolidada correspondiente al valor del Plan Anual de Adquisiciones para las Entidades Estatales.

\section{Tienda Virtual del Estado Colombiano}

- Grupo de datos que pone a disposición lista de precios de bienes y servicios y permite hacer órdenes de compra en línea. Estandariza precios para la compra pública.

\section{Multas y sanciones}

- Grupo de datos con la información consolidada de las multas y sanciones impuestas a proveedores.

Fuente: Colombia Compra Eficiente (2019)

Dentro de las plataformas SECOP, se puede descargar un registro histórico de procesos de compra pública, con diferentes opciones de filtro. Para el caso de proyectos de construcción está disponible la categoría "Terrenos, Edificios, Estructuras y Vías". Un esquema de la información disponible se presenta en la Figura 3. En la parte superior se reporta parte la información disponible y descargable de manera directa (en total son 62 columnas), que es la que evalúan las entidades internacionales. La información en la parte inferior de la figura corresponde a datos adicionales que pueden obtenerse si se descargan documentos del proyecto. 
SIBRAGEC - ELAGEC 2019 - del 23 al 25 de Octubre - LONDRINA - PR

Figura 3 - Información disponible en plataforma SECOP

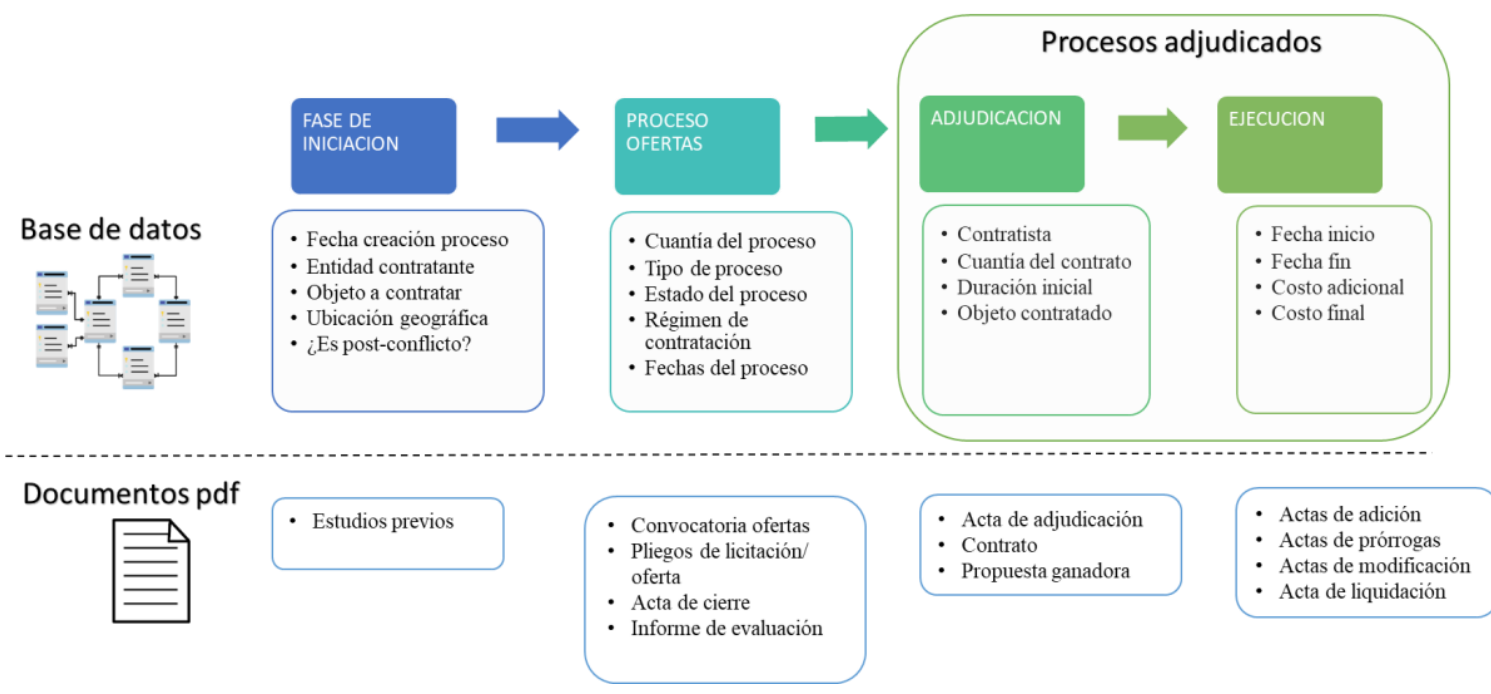

Fuente: Elaboración propia

\subsubsection{Ejemplo de análisis de datos}

Inicialmente se presenta un análisis de 126 proyectos de vías rurales ejecutados durante los años 2014-2018 y que fueron celebrados bajo la modalidad de licitación pública. La Figura 4 incluye los procesos con desviación en costos (izquierda) correspondiente al $60 \%$ de los casos y con desviación en tiempo (derecha) correspondiente al 56\% de los casos.

Figura 4 - Número de procesos con desviación en costo y tiempo

Procesos con/sin desviación en costo

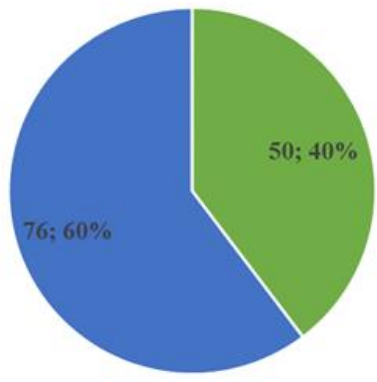

" Con desviación $\quad$ Sin desviación
Procesos con $/$ sin desviación en tiempo

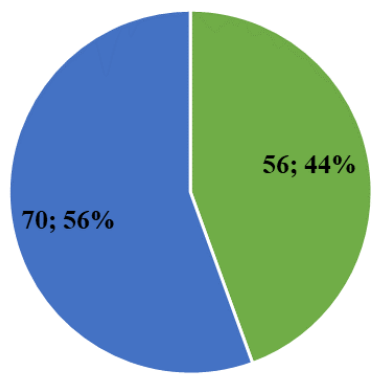

- Con desviación $\quad$ Sin desviación

Fuente: Elaboración propia

La Figura 5 incluye la magnitud de las desviaciones en costo (izquierda) y con desviación en tiempo (derecha). Cada uno de los puntos corresponde a un proceso y el eje vertical incluye la magnitud de la desviación en costo (Calculada en porcentaje). Es de anotar que la ley colombiana establece un máximo del $50 \%$ del valor inicial del contrato como límite máximo de costo adicional. La desviación en tiempo incluye valores desde cero hasta más de 3 veces la duración original del contrato. 
SIBRAGEC - ELAGEC 2019 - del 23 al 25 de Octubre - LONDRINA - PR

Figura 5 - Boxplot magnitud de las desviaciones en costo y tiempo.
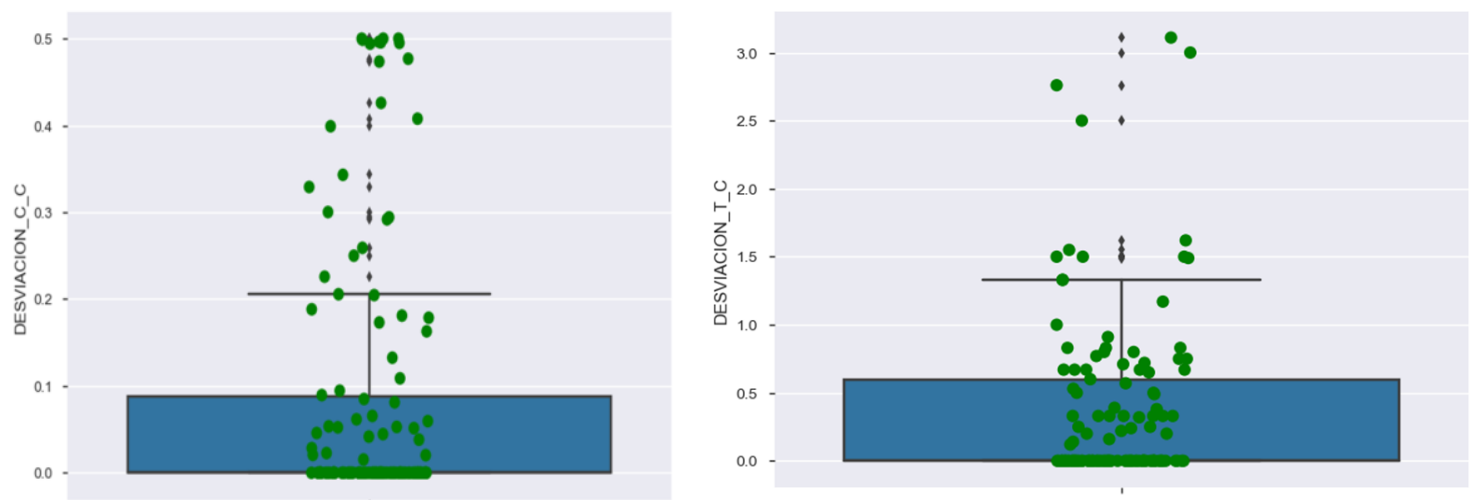

Fuente: Elaboración propia

Finalmente, la Figura 6 presenta el número de proponentes para los procesos de licitación, el cual es significativamente bajo. Siendo el proceso de licitación el más competitivo establecido en la legislación colombiana, llama la atención que en más del $75 \%$ de los procesos se haya presentado un único proponente.

\section{Figura 6 - Número de proponentes en procesos de licitación pública}

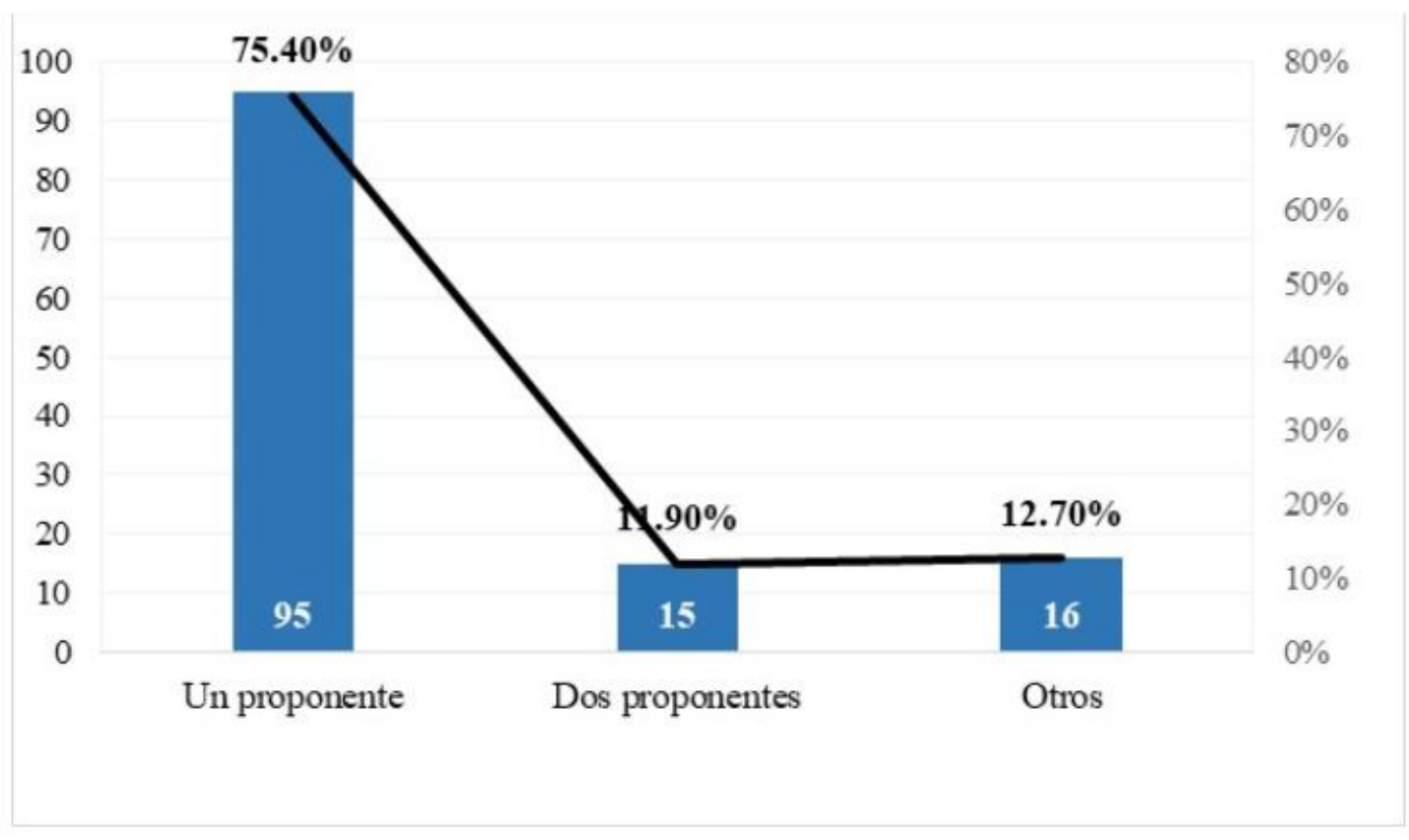

Fuente: Elaboración propia

\section{CONCLUSIONES}

La plataforma de compra pública del Estado Colombiano cuenta con datos de calidad, que brindan información información abierta, accesible y oportuna. Estos datos son descargables en formatos ampliamente utilizados, que permiten además diferentes opciones de filtro, facilitando el análisis. Esta información es libre y disponible en línea, por lo que cualquier ciudadano puede conocer el comportamiento del gasto 
público aumentando la transparencia en la ejecución y adicionalmente es posible realizar análisis que identifiquen tendencias que puedan aportar en la toma de decisiones.

La plataforma de datos abiertos del estado colombiano sigue los parámetros establecidos por iniciativas internacionales y también ha obtenido evaluaciones destacadas. Es destacable que se encuentre información desde las etapas tempranas del proyecto, su justificación, la información completa sobre la publicación y recibo de ofertas hasta la liquidación. Un aspecto por mejorar consiste en la información sobre los proponentes que se presentan en procesos de contratación, pues esta es significativa y aunque está disponible en la mayoría de procesos no es descargable de manera automática.

El caso de estudio de construcción de vías rurales presenta resultados desfavorables como un amplio número de procesos de licitación con único proponente y desviaciones en tiempo y costo en más de la mitad de los procesos de contratación. Estas tendencias deben ser estudiadas para la totalidad de los proyectos de construcción de obra pública y profundizar en las causas que generan este tipo de problemas.

Los datos abiertos también constituyen una eficaz herramienta contra la corrupción, pues es posible identificar los requisitos habilitantes en cada uno de los diferentes procesos, la duración de los procesos de contratación, los contratistas a quienes se adjudican, los valores con los que se está realizando la compra pública y compararlos además entre las diferentes entidades para así determinar la igualdad de condiciones para procesos similares.

\section{REFERENCIAS}

COLOMBIA COMPRA EFICIENTE. SECOP I | Colombia Compra Eficiente.

Disponível em: <https://www.colombiacompra.gov.co/compradores/secop-i>. Acesso em: 6 jul. 2018.

FAZEKAS, M.; KOCSIS, G. Uncovering High-Level Corruption: Cross-National Objective Corruption Risk Indicators Using Public Procurement Data. British Journal of Political Science, p. 1-10, 24 ago. 2017.

FAZEKAS, M.; TÓTH, I. J.; KING, L. P. An Objective Corruption Risk Index Using Public Procurement Data. European Journal on Criminal Policy and Research, v. 22, n. 3, p. 369-397, 2016.

FÖLSZ, H. et al. Data publication practices of public procurement authorities around the world $\mathbf{- 2 0 1 8}$. Budapest: [s.n.].

OCP, O. C. P. Open Contracting Data Standard: Documentation - OCDS 1.1.3 documentation. Disponível em: <http://standard.open-contracting.org/latest/en/>. Acesso em: 21 fev. 2019.

OPEN CONTRACTING PARTNERSHIP. Mythbusting Confidentiality In Public Contracting. [s.l: s.n.]. Disponível em: <https://www.open-contracting.org/wpcontent/uploads/2018/07/OCP18-Mythbusting.pdf>. Acesso em: 5 mar. 2019.

OPEN DATA CHARTER. The International Open Data Charter. Disponível em: <https://opendatacharter.net/>. Acesso em: 4 abr. 2019.

OPEN KNOWLEDGE INTERNATIONAL. Procurement - Global Open Data Index. Disponível em: <https://index.okfn.org/dataset/procurement/>. Acesso em: 26 abr. 
2019.

OWUSU, E. K. et al. An Empirical Study on Construction Process Corruption Susceptibility: A Vignette of International Expertise. Science and Engineering Ethics, 13 mar. 2019.

TRANSPARENCY INTERNATIONAL. Corruption Perceptions Index 2018 Transparency International. Disponível em:

<https://www.transparency.org/cpi2018>. Acesso em: 22 abr. 2019.

WORLD WIDE WEB FOUNDATION. Open Data Barometer.

ZULETA, M. M. Hacia una política de datos abiertos del Sistema de Compra Pública para los países miembros de la RICGOCDE Revisiones de recursos escolares : Chile 2017. [s.l: s.n.].

ZULETA, M. M.; SAAVEDRA, V.; MEDELLÍN, J. C. Fortalecimiento del sistema de compra pública para reducir el riesgo de corrupción. BOGOTA: [s.n.]. Disponível em: <https://www.repository.fedesarrollo.org.co/handle/11445/3544>. 